\title{
Real-world comparison of major bleeding risk among non-valvular atrial fibrillation patients initiated on apixaban, dabigatran, rivaroxaban, or warfarin
}

\section{A propensity score matched analysis}

\author{
Gregory Y. H. Lip ${ }^{1,2}$; Allison Keshishian3; Shital Kamble4; Xianying Pan; Jack Mardekian5; Ruslan Horblyuk; Melissa Hamilton ${ }^{4}$ \\ ${ }^{1}$ University of Birmingham Institute of Cardiovascular Sciences, City Hospital, Birmingham, UK; ${ }^{2}$ Aalborg Thrombosis Research Unit, Department of Clinical Medicine, Aalborg \\ University, Aalborg, Denmark; ${ }^{3}$ STATinMED Research, Ann Arbor, Michigan, USA; ${ }^{4}$ Bristol-Myers Squibb Company, Princeton, New Jersey, USA; ${ }^{5}$ Pfizer, Inc., New York, New York, USA
}

\begin{abstract}
Summary
In addition to warfarin, there are four non-vitamin $\mathrm{K}$ antagonist oral anticoagulants (NOACs) available for stroke prevention in non valvular atrial fibrillation (NVAF). There are limited data on the comparative risks of major bleeding among newly anticoagulated NVAF patients who initiate warfarin, apixaban, dabigatran, or rivaroxaban, when used in 'real world' clinical practice. The study used the Truven MarketScan ${ }^{\circledR}$ Commercial \& Medicare supplemental US claims database. NVAF patients aged $\geq 18$ years newly prescribed an oral anticoagulant 01JAN2013-31DEC2014, with a $\geq 1$-year baseline period, were included (study period: 01JAN2012-31DEC2014). Major bleeding was defined as bleeding requiring hospitalisation. Propensity score matching (PSM) was used to balance age, sex, region, baseline comorbidities, and comedications. Cox proportional hazards models were used to estimate the PSM hazard ratio (HR) of major bleeding. Among 45,361 newly anticoagulated NVAF patients, 15,461 (34.1\%) initiated warfarin, 7,438 (16.4\%) initiated apixaban, 17,801 (39.2\%) initiated rivaroxaban, and 4,661 (10.3\%) initiated dabigatran. Compared to
\end{abstract}

\section{Correspondence to:}

Prof. Gregory Y. H. Lip

University of Birmingham Institute of Cardiovascular Sciences

City Hospital, Birmingham, United Kingdom

Tel.: +44 121 5075080, Fax: +44 1215544083

E-mail: g.y.h.lip@bham.ac.uk

Note: The review process for this paper was fully handled by Christian

Weber, Editor in Chief. matched warfarin initiators, apixaban (HR: $0.53 ; 95 \% \mathrm{Cl}: 0.39-0.71$ ) and dabigatran (HR: $0.69 ; 95 \% \mathrm{Cl}: 0.50-0.96)$ initiators had a significantly lower risk of major bleeding. Patients initiating rivaroxaban (HR: $0.98 ; 95 \% \mathrm{Cl}: 0.83-1.17)$ had a non-significant difference in major bleeding risk compared to matched warfarin patients. When comparisons were made between NOACs, matched rivaroxaban patients had a significantly higher risk of major bleeding (HR: $1.82 ; 95 \% \mathrm{Cl}$ : 1.36-2.43) compared to apixaban patients. The differences for apixaban-dabigatran and dabigatran-rivaroxaban matched cohorts were not statistically significant. Among newly anticoagulated NVAF patients in the real-world setting, apixaban and dabigatran initiation was associated with significantly lower risk of major bleeding compared to warfarin initiation. When compared to apixaban, rivaroxaban initiation was associated with significantly higher risk of major bleeding.

\section{Keywords}

Non-valvular atrial fibrillation, major bleeding, warfarin, apixaban, dabigatran, rivaroxaban

\section{Received: May 25, 2016}

Accepted after major revision: July 27, 2016

Epub ahead of print: August 19, 2016

http://dx.doi.org/10.1160/TH16-05-0403

Thromb Haemost 2016; 116: 975-986

Supplementary Material to this article is available online at www.thrombosis-online.com.

\section{Introduction}

Atrial fibrillation (AF) increases the risk of ischaemic stroke by five-fold and is associated with $15 \%$ of strokes for all age groups and $30 \%$ in persons over the age of 80 years (1). AF-related stroke patients have higher recurrent risk, morbidity, and mortality as compared to patients with other stroke types (2). Current guidelines emphasise stroke prevention in AF patients, in the presence of stroke risk factors (3).

Effective stroke prevention essentially refers to oral anticoagulation $(\mathrm{OAC}$ ), and until recently, vitamin $\mathrm{K}$ antagonists (VKAs, e.g. warfarin) were the only OACs available in the United States.
The use of VKAs reduces stroke/systemic embolism by $64 \%$ and all-cause mortality by $26 \%$, compared to control or placebo (4). However, the use of VKAs has many limitations, including the need to ensure proper anticoagulation control by regular monitoring, given VKAs' many interactions with drugs and diet (5). As a result, $30-50 \%$ of AF patients were undertreated (6).

To overcome the limitations of VKAs, the non-VKA oral anticoagulants (NOACs) have been introduced and offer relative efficacy, safety, and convenience compared to VKA therapy (7). The available NOACs, dabigatran, rivaroxaban, apixaban, and edoxaban were approved by the US Food and Drug Administration in October 2010, November 2011, December 2013, and 
January 2015, respectively. In clinical trials, NOACs demonstrated similar or superior reduction in stroke and systemic embolism when compared to warfarin (7). Also, NOACs do not require routine INR blood monitoring and have fewer food and drug interactions (7).

The availability of warfarin and four NOACs in real-world clinical practice allows opportunities for comparative effectiveness analyses, particularly of the safety of these drugs when used outside the controlled setting of clinical trials. As edoxaban was only recently introduced to the market, and because little real-world data are available, this analysis only focused on warfarin, dabigatran, rivaroxaban and apixaban.

In the present study, our objective was to compare the risk of major bleeding among newly-anticoagulated non-valvular AF (NVAF) patients initiating on warfarin, rivaroxaban, dabigatran, or apixaban using propensity score matching (PSM). PSM was used to ensure comparability of patient populations in relation to outcomes when OACs were compared.

\section{Methods}

\section{Study design and data source}

A retrospective cohort study was conducted using the Truven MarketScan ${ }^{\circledast}$ Commercial Claims and Encounter and Medicare Supplemental and Coordination of Benefits Databases from January 2012 to December 2014 to compare major bleeding risk among NVAF patients. The MarketScan database is a high-quality resource with the combined claims of employer- and health plan-sourced data containing medical and drug data for several million individuals annually allowing for comprehensive longitudinal analysis. It is geographically representative of the commercially insured population in the United States. Furthermore, Medicare-eligible retirees with employer-sponsored private health insurance and employer-provided Medicare Supplemental plans in the United States are included. Medical information was obtained through the diagnosis codes in the claims from the International Classification of Diseases, Ninth Revision, Clinical Modification (ICD-9-CM). Pharmacy claims include the drug dispensed using the National Drug Code (NDC) coding system.

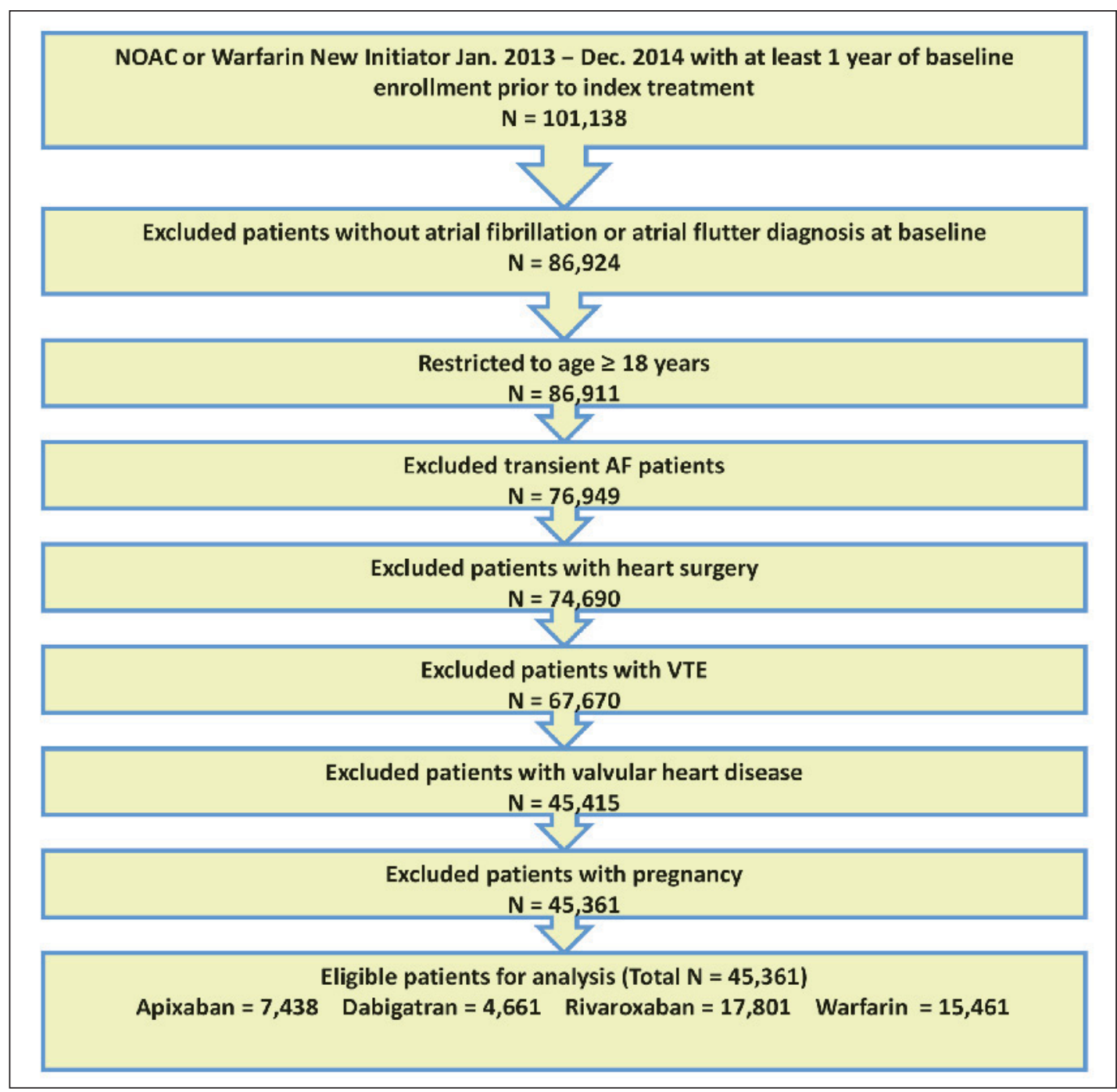

Thrombosis and Haemostasis 116.5/2016
Figure 1: Patient selection criteria. NOAC: Nonvitamin $\mathrm{K}$ antagonist oral anticoagulant; AF: atrial fibrillation; VTE: venous thromboembolism. 


\section{Selection criteria}

AF patients (ICD-9-CM codes: 427.31 or 427.32 ) $\geq 18$ years who newly initiated OACs (warfarin, dabigatran, rivaroxaban, and apixaban) from January 1, 2013 to December 31, 2014 were included in the study. The first OAC pharmacy claim date was designated as the index date. Patients with continuous health plan enrollment with medical and pharmacy benefits for at least 12 months preindex date (baseline period) were included in the study. Patients with a prescription claim for warfarin, rivaroxaban, dabigatran, or apixaban prior to the index date were excluded. Patients with evidence of transient AF (thyrotoxicosis, pericarditis), cardiac

Table 1: Baseline characteristics and treatment follow-up period for warfarin-NOAC propensity score matched cohorts.

\begin{tabular}{|c|c|c|c|c|c|c|c|c|c|c|c|c|}
\hline & \multicolumn{4}{|c|}{ Warfarin - Apixaban Cohort } & \multicolumn{4}{|c|}{ Warfarin-Dabigatran Cohort } & \multicolumn{4}{|c|}{ Warfarin-Rivaroxaban Cohort } \\
\hline & \multicolumn{2}{|l|}{ Warfarin } & \multicolumn{2}{|c|}{ Apixaban } & \multicolumn{2}{|l|}{ Warfarin } & \multicolumn{2}{|c|}{ Dabigatran } & \multicolumn{2}{|l|}{ Warfarin } & \multicolumn{2}{|c|}{ Rivaroxaban } \\
\hline & \multicolumn{2}{|c|}{$(N=6,964)$} & \multicolumn{2}{|c|}{$(N=6,964)$} & \multicolumn{2}{|c|}{$(\mathrm{N}=4,515)$} & \multicolumn{2}{|c|}{$(N=4,515)$} & \multicolumn{2}{|c|}{$(N=12,625)$} & \multicolumn{2}{|c|}{$(N=12,625)$} \\
\hline & Mean/N & $\mathrm{SD} / \%$ & Mean/N & SD $/ \%$ & Mean/N & $\mathrm{SD} / \%$ & Mean/N & SD $/ \%$ & Mean/N & $\mathrm{SD} / \%$ & Mean/N & $\mathrm{SD} / \%$ \\
\hline Age & 69.0 & 12.3 & 69.1 & 12.3 & 67.5 & 12.3 & 66.9 & 12.2 & 70.1 & 12.0 & 69.7 & 11.9 \\
\hline \multicolumn{13}{|l|}{ Gender } \\
\hline Male & 4,288 & $61.6 \%$ & 4,248 & $61.0 \%$ & 2,884 & $63.9 \%$ & 2,900 & $64.2 \%$ & 7,720 & $61.2 \%$ & 7,693 & $60.9 \%$ \\
\hline Female & 2,676 & $38.4 \%$ & 2,716 & $39.0 \%$ & 1,631 & $36.1 \%$ & 1,615 & $35.8 \%$ & 4,905 & $38.9 \%$ & 4,932 & $39.1 \%$ \\
\hline \multicolumn{13}{|l|}{ Region } \\
\hline Northeast & 1,419 & $20.4 \%$ & 1,419 & $20.4 \%$ & 1,018 & $22.6 \%$ & 1,030 & $22.8 \%$ & 2,426 & $19.2 \%$ & 2,447 & $19.4 \%$ \\
\hline North Central & 2,025 & $29.1 \%$ & 2,014 & $28.9 \%$ & 1,304 & $28.9 \%$ & 1,289 & $28.6 \%$ & 4,079 & $32.3 \%$ & 4,105 & $32.5 \%$ \\
\hline South & 2,342 & $33.6 \%$ & 2,405 & $34.5 \%$ & 1,350 & $29.9 \%$ & 1,359 & $30.1 \%$ & 3,428 & $27.2 \%$ & 3,395 & $26.9 \%$ \\
\hline West & 1,075 & $15.4 \%$ & 1,031 & $14.8 \%$ & 801 & $17.7 \%$ & 785 & $17.4 \%$ & 2,566 & $20.3 \%$ & 2,559 & $20.3 \%$ \\
\hline Other & 103 & $1.5 \%$ & 95 & $1.4 \%$ & 42 & $0.9 \%$ & 52 & $1.2 \%$ & 126 & $1.0 \%$ & 119 & $0.9 \%$ \\
\hline \multicolumn{13}{|l|}{ Baseline Comorbidity } \\
\hline $\begin{array}{l}\text { Deyo-Charlson Comorbidity } \\
\text { Index score }\end{array}$ & 1.8 & 2.0 & 1.9 & 2.0 & 1.6 & 1.9 & 1.6 & 1.9 & 2.0 & 2.1 & 2.0 & 2.1 \\
\hline $\mathrm{CHADS}_{2}$ Score & 1.8 & 1.2 & 1.8 & 1.2 & 1.6 & 1.2 & 1.6 & 1.2 & 1.9 & 1.2 & 1.8 & 1.2 \\
\hline $\mathrm{CHA}_{2} \mathrm{DS}_{2}$-VASc Score & 2.8 & 1.6 & 2.9 & 1.7 & 2.6 & 1.7 & 2.6 & 1.7 & 3.0 & 1.6 & 2.9 & 1.7 \\
\hline Modified HAS-BLED & 2.2 & 1.2 & 2.2 & 1.3 & 2.0 & 1.2 & 2.0 & 1.2 & 2.2 & 1.2 & 2.2 & 1.2 \\
\hline Baseline Prior Bleed & 962 & $13.8 \%$ & 980 & $14.1 \%$ & 523 & $11.6 \%$ & 536 & $11.9 \%$ & 2,017 & $16.0 \%$ & 1,976 & $15.7 \%$ \\
\hline Congestive Heart Failure & 1,371 & $19.7 \%$ & 1,398 & $20.1 \%$ & 852 & $18.9 \%$ & 864 & $19.1 \%$ & 2,783 & $22.0 \%$ & 2,792 & $22.1 \%$ \\
\hline Diabetes & 1,984 & $28.5 \%$ & 2,007 & $28.8 \%$ & 1,193 & $26.4 \%$ & 1,247 & $27.6 \%$ & 3,814 & $30.2 \%$ & 3,769 & $29.9 \%$ \\
\hline Hypertension & 5,139 & $73.8 \%$ & 5,173 & $74.3 \%$ & 3,148 & $69.7 \%$ & 3,150 & $69.8 \%$ & 9,128 & $72.3 \%$ & 9,099 & $72.1 \%$ \\
\hline Renal Disease & 653 & $9.4 \%$ & 628 & $9.0 \%$ & 348 & $7.7 \%$ & 333 & $7.4 \%$ & 1,342 & $10.6 \%$ & 1,292 & $10.2 \%$ \\
\hline Myocardial Infarction & 467 & $6.7 \%$ & 455 & $6.5 \%$ & 266 & $5.9 \%$ & 251 & $5.6 \%$ & 921 & $7.3 \%$ & 933 & $7.4 \%$ \\
\hline $\begin{array}{l}\text { Dyspepsia or Stomach } \\
\text { Discomfort }\end{array}$ & 952 & $13.7 \%$ & 1,026 & $14.7 \%$ & 534 & $11.8 \%$ & 560 & $12.4 \%$ & 1,868 & $14.8 \%$ & 1,886 & $14.9 \%$ \\
\hline Transient Ischaemic Attack & 377 & $5.4 \%$ & 373 & $5.4 \%$ & 170 & $3.8 \%$ & 202 & $4.5 \%$ & 663 & $5.25 \%$ & 645 & $5.11 \%$ \\
\hline Coronary Artery Disease & 2,203 & $31.6 \%$ & 2,269 & $32.6 \%$ & 1,209 & $26.8 \%$ & 1,266 & $28.0 \%$ & 4,052 & $32.1 \%$ & 4,036 & $32.0 \%$ \\
\hline Ischaemic Stroke & 545 & $7.8 \%$ & 586 & $8.4 \%$ & 297 & $6.6 \%$ & 318 & $7.0 \%$ & 1,169 & $9.3 \%$ & 1,126 & $8.9 \%$ \\
\hline \multicolumn{13}{|l|}{ Treatment Follow-up (in days) } \\
\hline Mean & 161.6 & 159.0 & 148.1 & 138.0 & 160.5 & 159.7 & 178.1 & 179.3 & 162.7 & 160.8 & 177.9 & 171.5 \\
\hline Median & 100.0 & & 96.0 & & 97.0 & & 100.0 & & 100.0 & & 113.0 & \\
\hline
\end{tabular}

$\mathrm{CHADS}_{2}$ : Congestive heart failure, Hypertension, Age $\geq 75$ years, Diabetes mellitus, prior Stroke, transient ischaemic attack or venous thromboembolism; $\mathrm{CHA}_{2} \mathrm{DS}_{2}$ VASC: CHADS 2 plus Vascular disease, Age 65-74 years, Sex category; HAS-BLED: Hypertension, Abnormal renal/liver dysfunction, Stroke, Bleeding history, Labile INR, Elderly, Drugs; SD: standard deviation. 
surgery, venous thromboembolism (VTE), valvular heart disease, or pregnancy were excluded ( Figure 1; ICD-9-CM codes are provided in Suppl. Table 1, available online at www.thrombosisonline.com). Patients were categorised into warfarin, dabigatran, rivaroxaban, or apixaban cohorts based on their first pharmacy claim. Patients were followed until their first major bleeding event, discontinuation of treatment, treatment switch, inpatient death, end of study period (December 31, 2014), 1 year after the index treatment date, or interruption in continuous health plan enrollment, whichever occurred earliest.

Table 2: Baseline characteristics and treatment follow-up period for NOAC-NOAC propensity score matched cohorts.

\begin{tabular}{|c|c|c|c|c|c|c|c|c|c|c|c|c|}
\hline & \multicolumn{4}{|c|}{ Apixaban - Dabigatran Cohort } & \multicolumn{4}{|c|}{ Apixaban - Rivaroxaban Cohort } & \multicolumn{4}{|c|}{ Dabigatran - Rivaroxaban Cohort } \\
\hline & \multirow{2}{*}{\multicolumn{2}{|c|}{$\begin{array}{l}\text { Apixaban } \\
(\mathrm{N}=4,407) \\
\end{array}$}} & \multirow{2}{*}{\multicolumn{2}{|c|}{$\begin{array}{l}\text { Dabigatran } \\
(\mathrm{N}=4,407)\end{array}$}} & \multirow{2}{*}{\multicolumn{2}{|c|}{\begin{tabular}{|l} 
Apixaban \\
$(\mathrm{N}=7,399)$ \\
\end{tabular}}} & \multirow{2}{*}{\multicolumn{2}{|c|}{$\begin{array}{l}\text { Rivaroxaban } \\
(\mathrm{N}=7,399) \\
\end{array}$}} & \multirow{2}{*}{\multicolumn{2}{|c|}{\begin{tabular}{|l|} 
Dabigatran \\
$(\mathrm{N}=4,657)$ \\
\end{tabular}}} & \multirow{2}{*}{\multicolumn{2}{|c|}{$\begin{array}{l}\text { Rivaroxaban } \\
(\mathrm{N}=4,657)\end{array}$}} \\
\hline & & & & & & & & & & & & \\
\hline & Mean/N & $\mathrm{SD} / \%$ & Mean/N & $\mathrm{SD} / \%$ & Mean/N & $\mathrm{SD} / \%$ & Mean/N & $\mathrm{SD} / \%$ & Mean/N & $\mathrm{SD} / \%$ & Mean/N & $\mathrm{SD} / \%$ \\
\hline Age & 67.0 & 12.3 & 66.9 & 12.2 & 68.4 & 12.4 & 68.3 & 12.2 & 66.5 & 12.4 & 66.3 & 12.3 \\
\hline \multicolumn{13}{|l|}{ Gender } \\
\hline Male & 2,823 & $64.1 \%$ & 2,822 & $64.0 \%$ & 4,549 & $61.5 \%$ & 4,550 & $61.5 \%$ & 3,026 & $65.0 \%$ & 2,965 & $63.7 \%$ \\
\hline Female & 1,584 & $35.9 \%$ & 1,585 & $36.0 \%$ & 2,850 & $38.5 \%$ & 2,849 & $38.5 \%$ & 1,631 & $35.0 \%$ & 1,692 & $36.3 \%$ \\
\hline \multicolumn{13}{|l|}{ Region } \\
\hline Northeast & 961 & $21.8 \%$ & 959 & $21.8 \%$ & 1,443 & $19.5 \%$ & 1,444 & $19.5 \%$ & 1,089 & $23.4 \%$ & 1,063 & $22.8 \%$ \\
\hline North Central & 1,238 & $28.1 \%$ & 1,251 & $28.4 \%$ & 2,035 & $27.5 \%$ & 2,044 & $27.6 \%$ & 1,300 & $27.9 \%$ & 1,313 & $28.2 \%$ \\
\hline South & 1,462 & $33.2 \%$ & 1,408 & $32.0 \%$ & 2,758 & $37.3 \%$ & 2,784 & $37.6 \%$ & 1,428 & $30.7 \%$ & 1,428 & $30.7 \%$ \\
\hline West & 701 & $15.9 \%$ & 736 & $16.7 \%$ & 1,040 & $14.1 \%$ & 994 & $13.4 \%$ & 786 & $16.9 \%$ & 799 & $17.2 \%$ \\
\hline Other & 45 & $1.0 \%$ & 53 & $1.2 \%$ & 123 & $1.7 \%$ & 133 & $1.8 \%$ & 54 & $1.2 \%$ & 54 & $1.2 \%$ \\
\hline \multicolumn{13}{|l|}{ Baseline Comorbidity } \\
\hline $\begin{array}{l}\text { Deyo-Charlson Comorbidity } \\
\text { Index score }\end{array}$ & 1.6 & 1.9 & 1.6 & 1.9 & 1.8 & 2.0 & 1.7 & 2.0 & 1.6 & 1.9 & 1.6 & 1.9 \\
\hline $\mathrm{CHADS}_{2}$ Score & 1.6 & 1.2 & 1.7 & 1.2 & 1.8 & 1.2 & 1.7 & 1.2 & 1.6 & 1.2 & 1.6 & 1.2 \\
\hline $\mathrm{CHA}_{2} \mathrm{DS}_{2}$-VASc Score & 2.5 & 1.6 & 2.6 & 1.7 & 2.8 & 1.6 & 2.8 & 1.7 & 2.5 & 1.7 & 2.5 & 1.6 \\
\hline Modified HAS-BLED & 2.0 & 1.2 & 2.0 & 1.2 & 2.2 & 1.2 & 2.1 & 1.2 & 2.0 & 1.2 & 1.9 & 1.2 \\
\hline Baseline Prior Bleed & 508 & $11.5 \%$ & 521 & $11.8 \%$ & 1,005 & $13.6 \%$ & 955 & $12.9 \%$ & 535 & $11.5 \%$ & 530 & $11.4 \%$ \\
\hline Congestive Heart Failure & 759 & $17.2 \%$ & 834 & $18.9 \%$ & 1,438 & $19.4 \%$ & 1,388 & $18.8 \%$ & 870 & $18.7 \%$ & 846 & $18.2 \%$ \\
\hline Diabetes & 1,190 & $27.0 \%$ & 1,203 & $27.3 \%$ & 2,095 & $28.3 \%$ & 2,021 & $27.3 \%$ & 1,269 & $27.3 \%$ & 1,313 & $28.2 \%$ \\
\hline Hypertension & 3,096 & $70.3 \%$ & 3,163 & $71.8 \%$ & 5,546 & $75.0 \%$ & 5,511 & $74.5 \%$ & 3,249 & $69.8 \%$ & 3,215 & $69.0 \%$ \\
\hline Renal Disease & 292 & $6.6 \%$ & 326 & $7.4 \%$ & 632 & $8.5 \%$ & 582 & $7.9 \%$ & 333 & $7.2 \%$ & 336 & $7.2 \%$ \\
\hline Myocardial Infarction & 226 & $5.1 \%$ & 246 & $5.6 \%$ & 465 & $6.3 \%$ & 452 & $6.1 \%$ & 251 & $5.4 \%$ & 282 & $6.1 \%$ \\
\hline $\begin{array}{l}\text { Dyspepsia or Stomach } \\
\text { Discomfort }\end{array}$ & 539 & $12.2 \%$ & 539 & $12.2 \%$ & 1,075 & $14.5 \%$ & 1,016 & $13.7 \%$ & 563 & $12.1 \%$ & 538 & $11.6 \%$ \\
\hline Transient Ischaemic Attack & 191 & $4.3 \%$ & 200 & $4.5 \%$ & 391 & $5.3 \%$ & 377 & $5.1 \%$ & 207 & $4.4 \%$ & 212 & $4.6 \%$ \\
\hline Coronary Artery Disease & 1,239 & $28.1 \%$ & 1,245 & $28.3 \%$ & 2,382 & $32.2 \%$ & 2,377 & $32.1 \%$ & 1,282 & $27.5 \%$ & 1,218 & $26.2 \%$ \\
\hline Ischaemic Stroke & 280 & $6.4 \%$ & 308 & $7.0 \%$ & 593 & $8.0 \%$ & 568 & $7.7 \%$ & 317 & $6.8 \%$ & 318 & $6.8 \%$ \\
\hline \multicolumn{13}{|l|}{ Treatment Follow-up (in days) } \\
\hline Mean & 145.6 & 136.5 & 179.0 & 179.1 & 147.6 & 137.6 & 182.1 & 174.9 & 177.3 & 178.7 & 172.5 & 169.5 \\
\hline Median & 93.0 & & 103.0 & & 95.0 & & 116.0 & & 100.0 & & 111.0 & \\
\hline
\end{tabular}

$\mathrm{CHADS}_{2}$ : Congestive heart failure, Hypertension, Age $\geq 75$ years, Diabetes mellitus, prior Stroke, transient ischaemic attack or venous thromboembolism; $\mathrm{CHA}_{2} \mathrm{DS}_{2}$ VASC: CHADS 2 plus Vascular disease, Age 65-74 years, Sex category; HAS-BLED: Hypertension, Abnormal renal/liver dysfunction, Stroke, Bleeding history, Labile INR, Elderly, Drugs; SD: standard deviation. 


\section{Outcome measures}

Major bleeding was defined as bleeding requiring hospitalisation during the period of drug use or within 30 days after the last days of supply of the treatment prescription. Major bleeding was identified using hospital claims, which had a bleeding diagnosis code as the first listed primary ICD-9 diagnosis code (Suppl. Table 1, available online at www.thrombosis-online.com). The definition of major bleeding

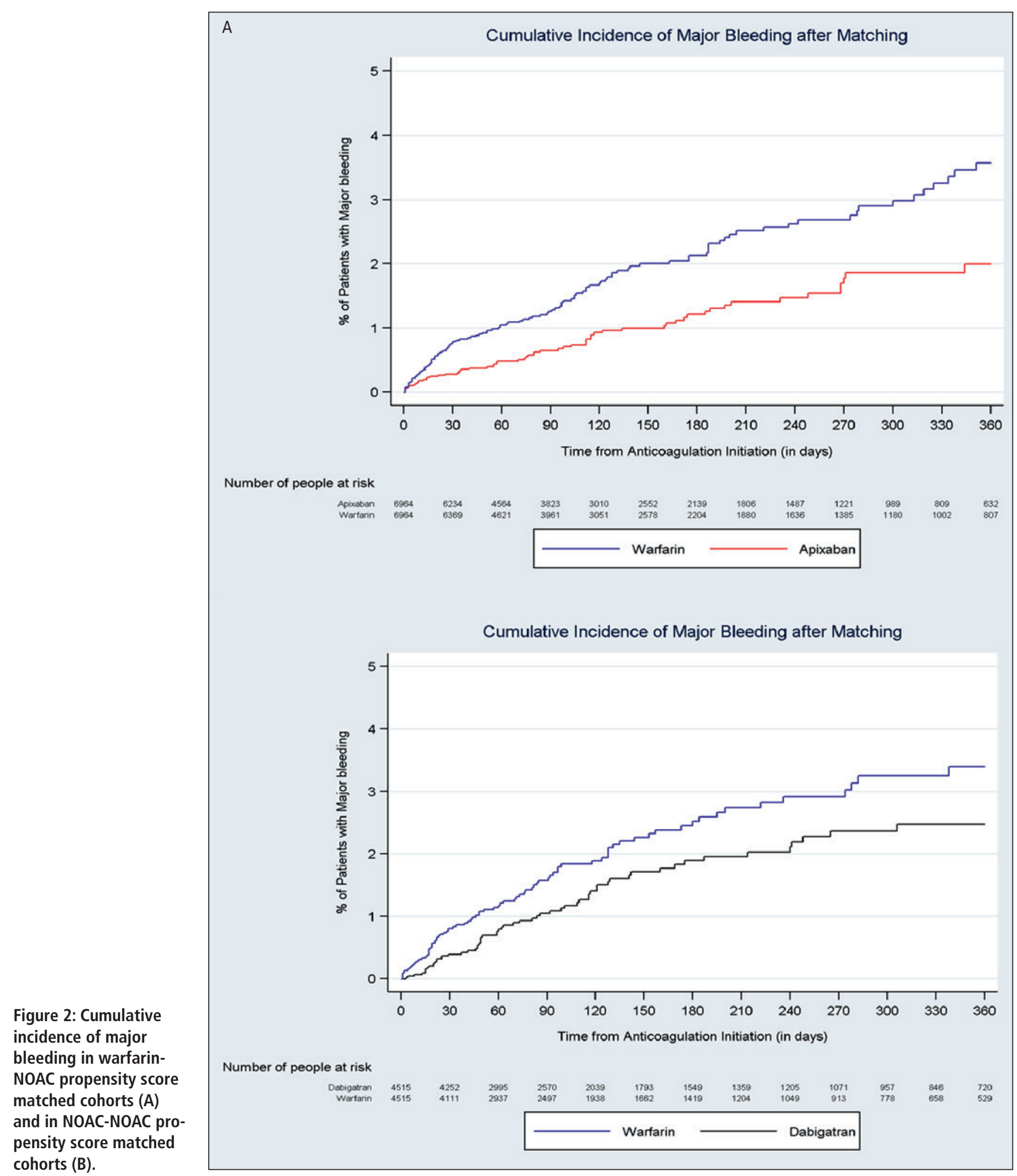




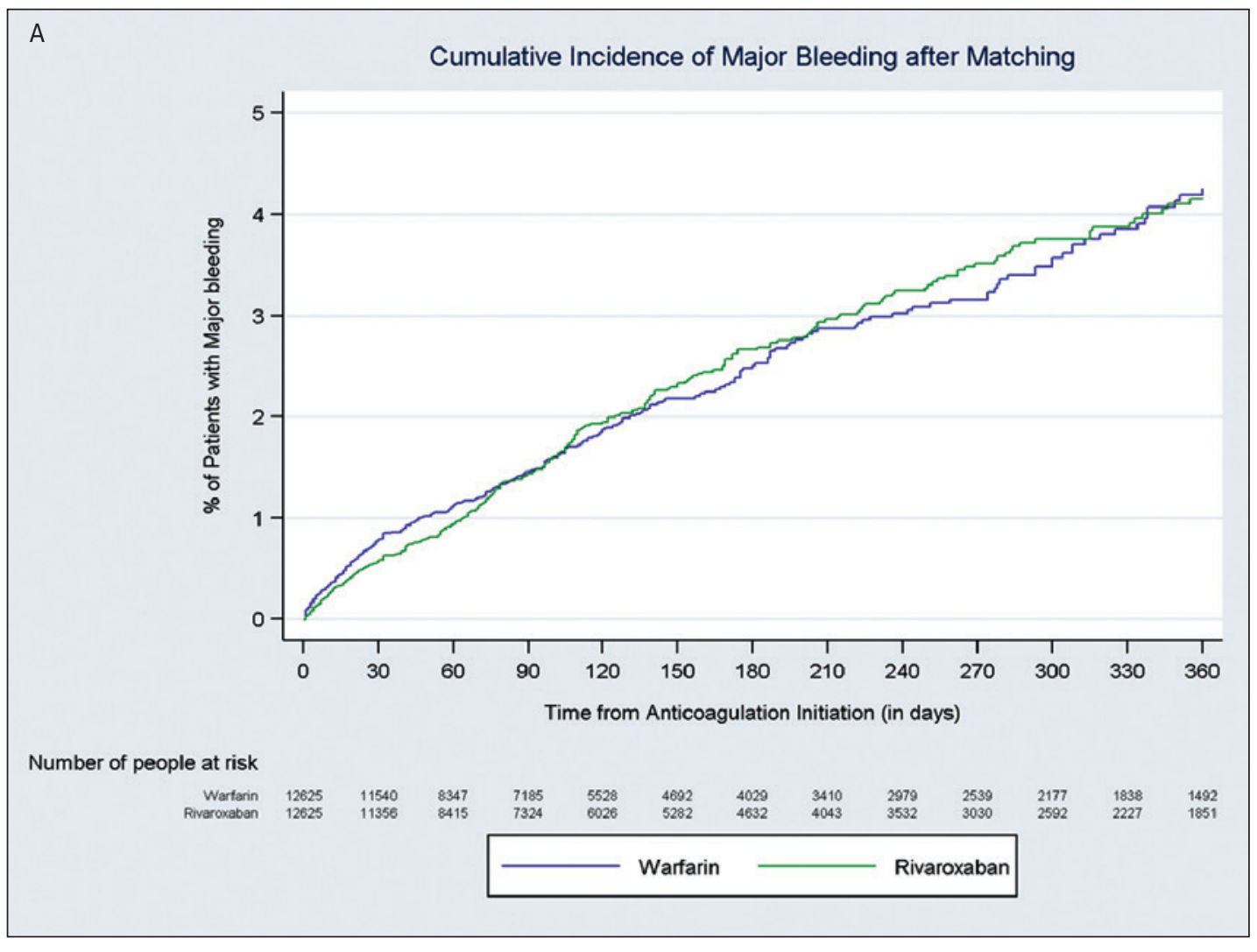

Figure 2A: Continued.

was based on a published administrative claims-based algorithm as well as clinical trial definitions of major bleeding $(8,9)$. This definition accounts for major bleeding at key sites including, but not limited to intracranial, gastrointestinal, liver, splenic, and ocular haemorrhage requiring hospitalisation with a diagnosis for bleeding.

\section{Statistical methods}

Pre-matched patient characteristics measured during the baseline period, including age, gender, geographic region, clinical characteristics, and baseline medication use were compared using Kruskal-Wallis and Chi-squared tests. Clinical characteristics were identified using ICD-9 codes and included, dyspepsia or stomach discomfort, congestive heart failure, coronary artery disease, diabetes, hypertension, renal disease (e.g. acute glomerulonephritis, nephrotic syndrome, and chronic and acute kidney disease), myocardial infarction, ischaemic stroke, transient ischaemic attack, history of bleeding, Deyo-Charlson Comorbidity Index (CCI) score, $\mathrm{CHADS}_{2}$ score, $\mathrm{CHA}_{2} \mathrm{DS}_{2}$-VASc score, and a (modified) HAS-BLED score. As the International Normalised Ratio (INR) is not available in the MarketScan database, a modified HAS-BLED score was calculated with a range of 0 to 8 .

To compare risk of major bleeding among NVAF patients who initiated OACs, PSM pair-wise comparisons were conducted between each cohort, matching NOACs to warfarin and also matching among NOACs. Propensity scores were estimated by uncondi- tional logistic regression that incorporated potential predictors of treatment as independent variables in the regression, and group status (e.g. apixaban initiators vs warfarin initiators), as the outcome (10). Sociodemographic and clinical characteristics that were associated with treatment and risk of major bleeding were included in the model to adjust for differences across cohorts. The same covariates were included in each matched population. A 1: 1 match was performed using the nearest neighbour technique without replacement with a maximum caliper of $0.01(11,12)$. Standardised mean differences were used to assess the balance of patient confounders at baseline. The value of absolute standardised mean difference $\leq 0.1$ indicates a negligible difference in potential confounders and balanced matched cohorts. The distribution of the propensity scores was reviewed for each matched population to ensure balance.

The incidence rate of major bleeding was calculated as the number of first major bleeding events divided by the total time at risk for major bleeding within the study period and described as the number of bleeding events per 100 person-years. The cumulative incidence of major bleeding was compared and presented using Kaplan-Meier curves. Cox proportional hazard models for the PSM matched cohorts were used to estimate the relative risk of major bleeding with $95 \%$ confidence intervals (CIs). A p-value of $<0.05$ was considered statistically significant. All analyses were conducted using SAS 9.3.

Sensitivity analysis was conducted to test the robustness of the study results. A dose-based interaction effect may be observed 
with major bleeding; therefore, we assessed the treatment effect associated with risk of major bleeding among patients prescribed the standard dose for all OACs (warfarin, apixaban $5 \mathrm{mg}$ BID, rivaroxaban $20 \mathrm{mg}$ QD, or dabigatran $150 \mathrm{mg}$ BID) (13).

\section{Results}

A total of 45,361 patients were identified after applying the study selection criteria, including 7,438 (16.40\%) patients in the apixaban cohort, $4,661(10.28 \%)$ in the dabigatran cohort, 17,801

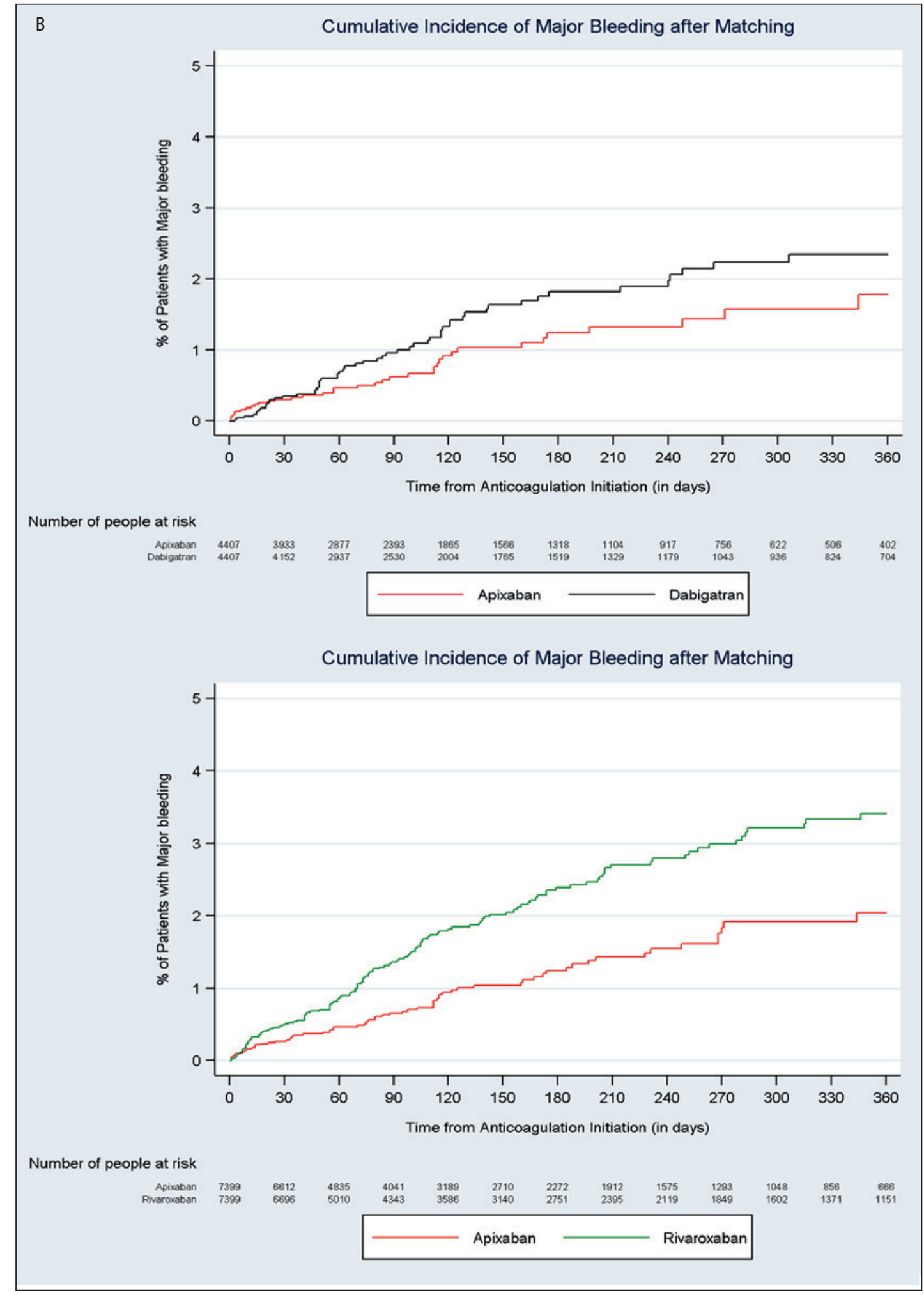

Figure 2B: Continued. 


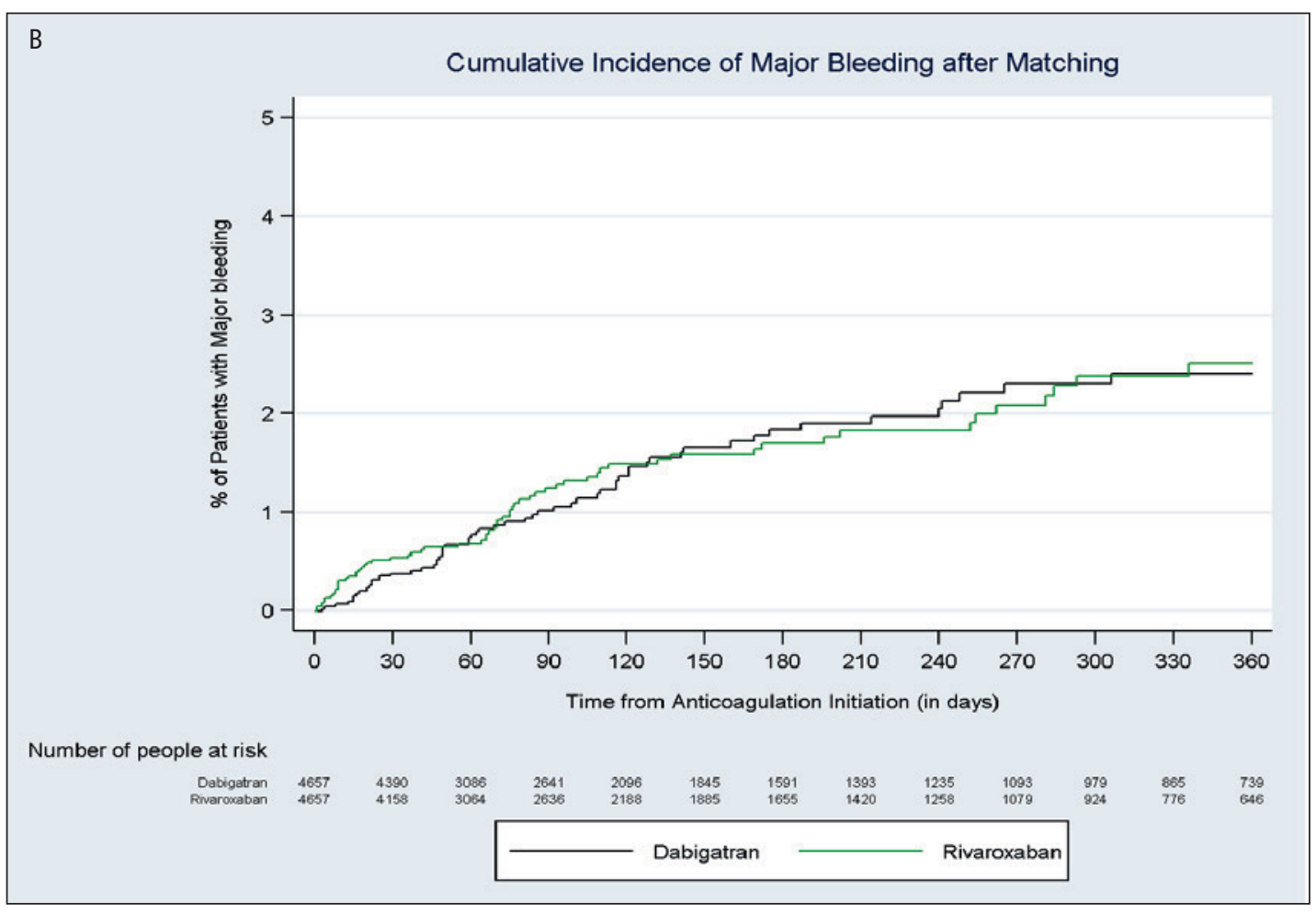

Figure 2B: Continued.

(39.24\%) in the rivaroxaban cohort, and 15,461 (34.08\%) in the warfarin cohort ( $>$ Figure 1$)$.

Pre-matched data showed that warfarin patients were generally older, had more comorbidities and higher risk profiles compared to NOAC users (all p-values $<0.001$; Suppl. Table 2, available online at www.thrombosis-online.com). There were 6,964 matched warfarin-apixaban patients, 4,515 matched warfarin-dabigatran patients, and 12,625 matched warfarin-rivaroxaban patients ( $>$ Table 1). When NOACs were matched between each other, there were 4,407 matched apixaban-dabigatran patients, 7,399 matched apixaban-rivaroxaban patients, and 4,657 matched dabigatran-rivaroxaban patients ( $>$ Table 2 ). Following PSM, the baseline demographics and clinical characteristics, including the risk scores $\left(\mathrm{CHA}_{2} \mathrm{DS}_{2}\right.$-VASc and modified HAS-BLED), were balanced with all standardised differences less than 0.1 between the matched cohorts.

Figure 2 shows the cumulative incidence of major bleeding in each matched cohort across the study period.

The incidence rates of major bleeding for NOAC and warfarin matched cohorts are shown in Figure $3 \mathrm{~A}$ with the lowest rate of 2.38 per 100 person-years observed among apixaban initiators in the apixaban-warfarin matched cohort, and the highest rate of 5.09 per 100 person-years for warfarin initiators in the warfarinrivaroxaban matched cohort ( Figure $3 \mathrm{~A}$ ). Cox proportional hazard models were then performed to compare the risk of major bleeding between warfarin and NOACs. Apixaban (hazard ratio [HR]: 0.53; 95\% CI: 0.39-0.71) and dabigatran (HR: 0.69; 95\% CI: $0.50-0.96)$ initiators had a significantly lower risk of major bleed- ing compared to matched warfarin initiators. Patients initiating rivaroxaban (HR: 0.98; $95 \%$ CI: 0.83-1.17) had a statistically nonsignificant difference in major bleeding risk compared to matched warfarin patients ( $>$ Figure $3 \mathrm{~A}$ ).

The incidence rates of major bleeding for NOAC to NOAC matched cohorts are shown in $>$ Figure $3 \mathrm{~B}$. The incidence rates ranged from 2.42 to 4.24 in the apixaban and rivaroxaban matched cohorts, respectively ( Figure $3 \mathrm{~B}$ ). When comparisons were made between NOACs, matched rivaroxaban patients had a significantly higher risk of major bleeding (HR: 1.82; $95 \% \mathrm{CI}$ : 1.36-2.43) compared to patients newly initiated on apixaban. The difference for dabigatran was not statistically significant (HR: 1.41; 95\% CI: 0.93-2.14) when compared to matched apixaban initiators. Patients initiating rivaroxaban (HR: 1.05; 95\% CI: 0.74-1.49) had a statistically non-significant difference in major bleeding risk compared to matched dabigatran initiators ( $>$ Figure 3B).

\section{Sensitivity analysis}

In the sensitivity analysis of patients on standard dose NOACs (apixaban $5 \mathrm{mg}$ BID, dabigatran $150 \mathrm{mg}$ BID, rivaroxaban $20 \mathrm{mg}$ QD) and warfarin, baseline characteristics were similar between all patients and patients on standard dose NOACs (Suppl. Tables 3-5, available online at www.thrombosis-online.com). Patients prescribed the low dose were not included in this analysis: $13.5 \%$ of patients were prescribed apixaban $2.5 \mathrm{mg}, 10.6 \%$ were prescribed dabigatran $75 \mathrm{mg}$, and $19.6 \%$ were prescribed rivaroxaban 


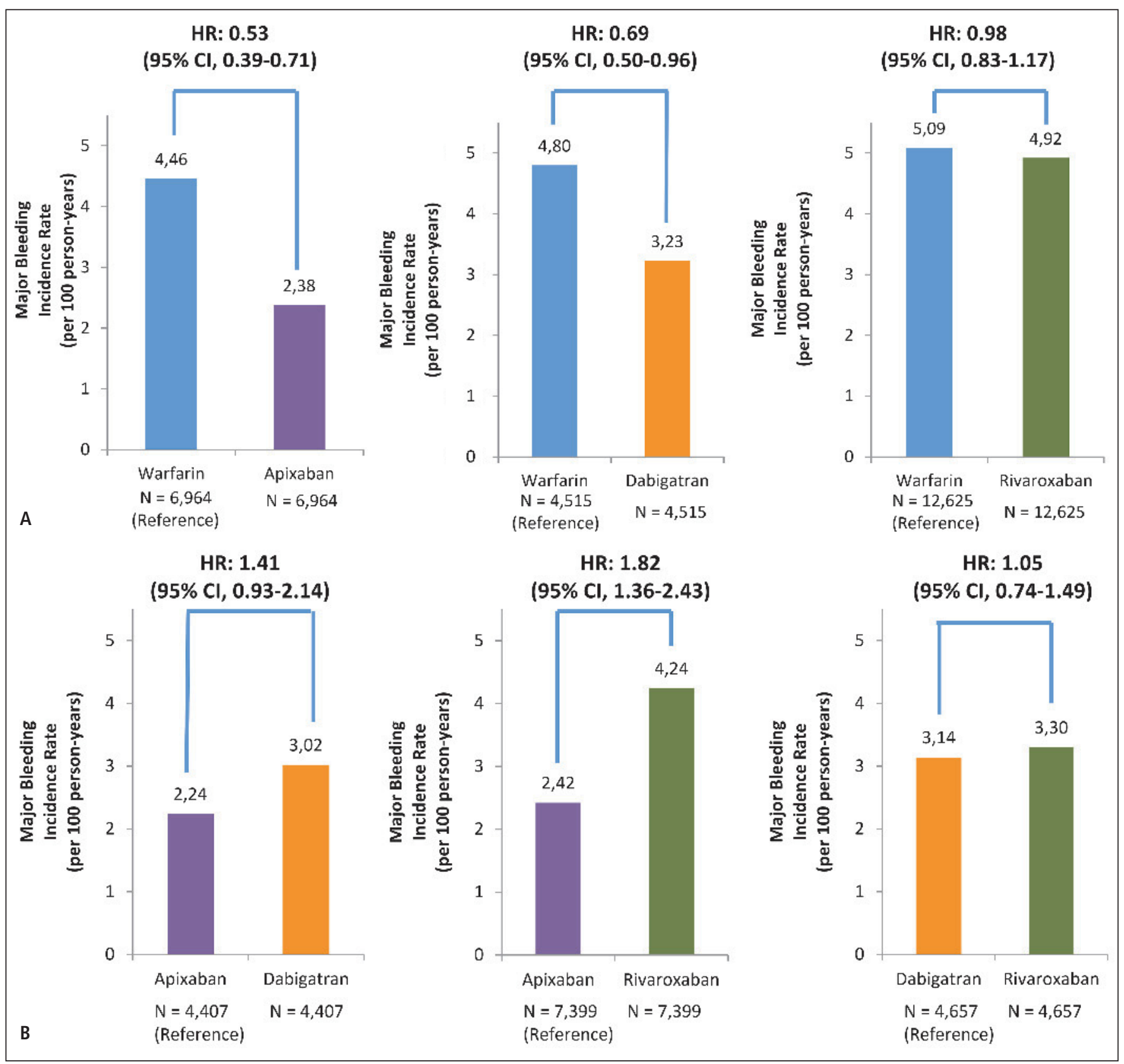

Figure 3: Major bleeding incidence rates and hazard ratios in warfarin-NOAC propensity score matched cohorts (A) and in NOAC-NOAC propensity score matched cohorts (B). Cl: confidence interval; HR: hazard ratio.

$15 \mathrm{mg}$. The PSM matched incidence rates of major bleeding were similar but slightly lower in the matched standard dose population (Suppl. Figures 1 and 2, available online at www.thrombosis-on line.com). Trends of major bleeding risk in the sensitivity analysis were found to be consistent to the main analyses, except that patients newly initiated on dabigatran $150 \mathrm{mg}$ had a non-significant difference in major bleeding risk versus matched warfarin patients (HR: $0.71 ; 95 \%$ CI: $0.49-1.05$ ) and patients newly initiated on rivaroxaban $20 \mathrm{mg}$ had a significantly higher risk of major bleeding versus matched dabigatran $150 \mathrm{mg}$ patients (HR: 1.65; $95 \% \mathrm{CI}$ :
1.15-2.36) (Suppl. Figures 1 and 2, available online at www. thrombosis-online.com).

\section{Discussion}

In this study, our principal findings are that given similar demographic and clinical characteristics with PSM, dabigatran and apixaban were associated with a significantly lower risk of major bleeding compared to warfarin, among newly anticoagulated 
NVAF patients. There was no significant difference in risk of major bleeding between matched rivaroxaban and warfarin initiators. When NOACs were compared, rivaroxaban initiation was associated with a significantly increased risk of major bleeding contrasted to apixaban initiation. There was no significant difference in risk of major bleeding when rivaroxaban initiators were compared to dabigatran initiators. The difference in risk of major bleeding for dabigatran initiators was not statistically significant when compared to matched apixaban initiators.

This is the one of the first PSM comparative safety studies assessing the risk of major bleeding among newly-initiated warfarin, dabigatran, rivaroxaban, and apixaban patients. By pair-wise comparisons, patients with similar demographic and clinical characteristics were matched and compared directly. This study also used the comprehensive Truven MarketScan claims database, which incorporates both medical and pharmacy claims of patients in the United States and allows generalizability among the commerciallyinsured and Medicare populations. We also found it reassuring that the (modified) HAS-BLED scores were balanced across all matched cohorts, reflecting comparability in baseline bleeding risk to the extent measurable within this dataset.

These real-world observational data are consistent with clinical trial safety observations. Our study showed that in the matched cohorts, as compared to warfarin, patients who initiated dabigatran had significantly lower incidence rate and risk of major bleeding. In the RE-LY trial, the rate of major bleeding was $3.36 \%$ per year for warfarin, compared to $2.71 \%$ per year (HR: 0.80; $95 \%$ CI: $0.69-0.93$; $\mathrm{p}=0.003)$ for $110 \mathrm{mg}$ BID and $3.11 \%$ per year (HR: 0.93; 95\% CI: 0.81-1.07; p=0.31) for $150 \mathrm{mg}$ BID dabigatran (12). Moreover, in the sensitivity analysis of dabigatran $150 \mathrm{mg}$ BID initiators, there was no significant difference in risk of major bleeding compared to patients who initiated warfarin. In the ROCKET-AF trial, the rates of major bleeding were not significantly different between warfarin and rivaroxaban (HR: 1.03; $95 \%$ CI: 0.96-1.11) (14). Similar to the ROCKET-AF, our study did not find significant difference in risk and incidence of major bleeding between rivaroxaban and warfarin initiators.

In the ARISTOTLE trial, apixaban showed a significantly lower rate of major bleeding compared to warfarin (HR: 0.69; 95\% CI: 0.60-0.80).9 This trend was consistent in our real-world study, which showed a significantly lower incidence rate and risk of major bleeding among patients who initiated apixaban as compared to warfarin. As far as we are aware, no other US-based observational study has evaluated the risk of major bleeding between multiple NOACs and warfarin using PSM.

In general, real-world data have provided complementary evidence on the efficacy and safety of the NOACs compared to warfarin. Some real-world results regarding dabigatran and rivaroxaban use have been published with no comparator or only compared to warfarin. In a non-comparative international prospective observational study, patients treated with rivaroxaban had a major bleeding incidence of 2.1 events per 100 person-years (15). In respect to comparative real world data studies of major gastrointestinal bleeding between rivaroxaban and warfarin, the findings of this study are comparable, showing that risk of bleeding with riva- roxaban did not significantly differ from warfarin (16). Real-world data for dabigatran have shown similar or lower risk of major bleeding compared to warfarin, consistent with the present study $(15,17)$. For example, the FDA post-marketing report for dabigatran showed a lower risk of gastrointestinal and intracranial haemorrhage compared with warfarin (1.6 vs 3.5 events/100,000 days; 0.8 vs 2.4 events/100,000 days) (18). However, other realworld studies have shown a higher risk of major gastrointestinal bleeding among patients who initiated dabigatran compared to patients who initiated warfarin (19).

The present analysis adds to the results of prior clinical trials and real-world studies by comparing the safety of three of the available NOACs against warfarin in a US-based cohort, and provides insights into the relative safety of the NOACs compared to each other in an OAC inception cohort. Patients who initiated rivaroxaban were significantly more likely to have a major bleeding event compared to matched patients who initiated apixaban. While no randomised controlled trial results exist comparing NOACs, these findings are aligned with meta-analyses, based on clinical trials data, that apixaban is associated with significantly lower risk of major bleeding compared to warfarin and rivaroxaban (20). Another study of indirect comparisons between NOACs concluded that patients prescribed rivaroxaban had significant higher risk of major bleeding compared to dabigatran 110 mg BID, and apixaban showed significant lower risk of major bleeding compared to dabigatran $150 \mathrm{mg} \operatorname{BID}(19,21)$.

Importantly, sensitivity analyses confined to the standard dose NOACs only (apixaban $5 \mathrm{mg}$ BID, dabigatran $150 \mathrm{mg}$ BID, rivaroxaban $20 \mathrm{mg}$ QD) demonstrated similar trends for risk of major bleeding. However, when the analysis was limited to patients who initiated dabigatran $150 \mathrm{mg}$, no significant difference in risk of major bleeding compared to those who initiated warfarin was observed, whilst patients newly initiated on rivaroxaban $20 \mathrm{mg}$ had a significantly higher risk of major bleeding versus matched dabigatran patients. Our data are comparable and consistent with a recent propensity weighted analysis from the Danish nationwide cohort study comparing standard-dose NOACs and warfarin, where the risks of death, any bleeding, or major bleeding were significantly lower for apixaban and dabigatran compared with warfarin (22).

\section{Limitations}

Given the nature of retrospective observational study, only associations can be drawn from this study. As is the case with claims databases, there is a potential for coding errors and missing data. Permanent, persistent and paroxysmal AF cannot be differentiated using ICD-9 codes, which is a general limitation of retrospective database analysis of claims data. Although PSM minimizes the potential bias due to observed confounding, there may still be residual confounding due to unobserved confounders that cannot be measured in a claims database, such as over-the-counter aspirin use or incomplete claims. Given that patients were prescribed a particular agent depending on the physician in a nonrandomised manner, some possibility of selection bias is evident, as is the case 
for any observational study. The PSM was conducted between each of the two OAC cohorts, thus the results are not comparable across all matched populations. For example, the warfarindabigatran and apixaban-dabigatran matched cohorts were younger and had lower $\mathrm{CHA}_{2} \mathrm{DS}_{2}$-VASc scores compared to other matched populations, indicating better health conditions in these two cohorts and possibly lower incidence rates and risks of major bleeding. In addition, no adjustments were made for multiple comparisons.

We do not have data on the quality of anticoagulation control among warfarin users, as reflected by time in therapeutic range (TTR), which is important given the relationship of TTR to the efficacy and safety of VKA therapy (23). Additionally, we do not have laboratory results such as creatinine clearance or INR so we cannot control for their effects on the initiation of drugs. In addition, only inpatient deaths are observed and reasons for mortality are not available in the database, which may have biased the survival analysis. We conducted a sensitivity analysis using a subdistribution hazard model with the Fine and Gray test to evaluate death as a competing risk. The findings remained consistent with the main analysis and inpatient death did not have an impact on the results $(24,25)$. The mean length of follow-up for apixabantreated patients was approximately one month shorter than for the other OACs. We used survival methods to account for varying lengths of follow-up. However, if bleeding events tend to occur later on apixaban than the other OACs, the difference in follow-up period may have affected the results. Across all of the cohorts there is a relatively short follow-up period (4-6 months), which reflects the high discontinuation of anticoagulation observed in the realworld setting.

These data are also based on the United States healthcare system and may have limited generalisability outside the US. Dabigatran $110 \mathrm{mg}$ BID is not licensed in the US for stroke prevention in $\mathrm{AF}$, and the $75 \mathrm{mg}$ dose is only licensed for significant renal impairment (creatinine clearance 15-29 $\mathrm{ml} /$ minute) only in the US, and not in the rest of the world. Although Truven MarketScan database allows for the selection of a nationally representative sample for this study, the results may not be necessarily generalisable to the entire NVAF population in the United States. Further research is needed to compare the effectiveness and safety of NOACs and warfarin in clinical practice.

\section{Conclusion}

This 'real-world' observational study using propensity score matched cohorts demonstrates that apixaban and dabigatran initiation was associated with significantly lower risk of major bleeding compared to warfarin initiation among newly anticoagulated NVAF patients in the real world setting amongst US patients. When comparisons were made between NOACs, rivaroxaban initiation was associated with significantly higher risk of major bleeding compared to apixaban initiation.

\section{What is known about this topic?}

- In addition to warfarin, there are four non-vitamin K antagonist oral anticoagulants (NOACs) available for stroke prevention in non valvular atrial fibrillation (NVAF).

- There are limited data on the comparative risks of major bleeding among newly anticoagulated NVAF patients who initiate warfarin, apixaban, dabigatran, or rivaroxaban, when used in 'real world' clinical practice.

\section{What does this paper add?}

- Using propensity score matching (PSM), the risk of major bleeding among NVAF patients newly prescribed warfarin, dabigatran, apixaban, or rivaroxaban was compared.

- Compared to warfarin initiation, dabigatran and apixaban initiation demonstrated significantly lower risk of major bleeding. There was no significant difference in risk of major bleeding when matched rivaroxaban and warfarin patients were compared.

- When NOACs were compared, matched rivaroxaban patients had a significantly higher risk of major bleeding compared to patients newly initiated on apixaban. There was no statistically significant difference in risk of major bleeding between apixaban or rivaroxaban initiators matched to dabigatran initiators.

- This is one of the first PSM comparative safety studies assessing the risk of major bleeding among newly-initiated warfarin, dabigatran, rivaroxaban, and apixaban US-based patients.

\section{Conflicts of interest}

This work was supported by Bristol-Myers Squibb and Pfizer. Professor Lip has served as a consultant for Bayer, BMS/Pfizer, Daiichi-Sankyo, Biotronik, Medtronic and Boehringer Ingelheim and has been on the speaker's bureau for Bayer, BMS/Pfizer, Boehringer Ingelheim, Daiichi-Sankyo, Medtronic. Ms. Keshishian is an employee of STATinMED Research who was a paid contractor to Bristol-Myers Squibb and Pfizer in connection with conducting this study and with the development of this manuscript. Dr. Kamble is an employee of Bristol-Myers Squibb with ownership of stocks in Bristol-Myers Squibb. Ms. Pan and Ms. Hamilton are employees of Bristol-Myers Squibb with ownership of stocks in Bristol-Myers Squibb. Dr. Mardekian and Mr. Horblyuk are employees of Pfizer Inc. with ownership of stocks in Pfizer Inc. The authors have indicated that they have no other conflicts of interest regarding the content of this article.

\section{References}

1. Wolf PA, Abbott RD, Kannel WB. Atrial fibrillation: a major contributor to stroke in the elderly: the Framingham Study. Arch Intern Med 1987; 147: $1561-1564$

2. Savelieva I, Bajpai A, Camm AJ. Stroke in atrial fibrillation: update on pathophysiology, new antithrombotic therapies, and evolution of procedures and devices. Ann Med 2007; 39: 371-391. 
3. January CT, Wann LS, Alpert JS, et al. 2014 AHA/ACC/HRS guideline for the management of patients with atrial fibrillation. J Am Coll Cardiol 2014; 64: e1-76.

4. Hart RG, Pearce LA, Aguilar MM. Adjusted-dose warfarin versus aspirin for preventing stroke in patients with atrial fibrillation. Ann Intern Med 2007; 147: 590-592.

5. De Caterina R, Husted S, Wallentin L, et al. Vitamin K antagonists in heart disease: Current status and perspectives (Section III). Thromb Haemost 2013; 110: 1087-1107.

6. Ogilvie IM, Newton N, Welner SA, Cowell W, Lip GY. Underuse of oral anticoagulants in atrial fibrillation: a systematic review. Am J Med 2010; 123: 638-645.

7. Ruff CT, Giugliano RP, Braunwald E, et al. Comparison of the efficacy and safety of new oral anticoagulants with warfarin in patients with atrial fibrillation: a meta-analysis of randomised trials. Lancet 2014; 383: 955-962.

8. Cunningham A, Stein CM, Chung CP, et al.. An automated database case definition for serious bleeding related to oral anticoagulant use. Pharmacoepidemiol Drug Saf 2011; 20: 560-566.

9. Granger $\mathrm{CB}$, Alexander JH, McMurray JJ, et al. Apixaban versus warfarin in patients with atrial fibrillation. New Engl J Med 2011; 365: 981-992.

10. Pattanayak CW, Rubin DB, Zell ER. Propensity score methods for creating covariate balance in observational studies. Rev Esp Cardiol 2011; 6410: 897-903.

11. Baser O. Too Much Ado about Propensity Score Models? Comparing Methods of Propensity Score Matching. Value Health 2006; 9: 377-385.

12. Austin, PC. An introduction to propensity score methods for reducing the effects of confounding in observational studies. Behavioral Res 2011; 46: 399-424.

13. Connolly SJ, Ezekowitz MD, Yusuf S, et al. Dabigatran versus warfarin in patients with atrial fibrillation. New Engl J Med 2010; 361: 1139-1151.

14. Patel MR, Mahaffey KW, Garg J, et al. Rivaroxaban versus warfarin in nonvalvular atrial fibrillation. New Engl J Med 2011; 365: 883-891.
15. Camm AJ, Amarenco P, Haas S, et al. XANTUS: a real-world, prospective, observational study of patients treated with rivaroxaban for stroke prevention in atrial fibrillation. Eur Heart J 2016; 37: 1145-1153.

16. Abraham NS, Singh S, Akexander GC et al. Comparative Risk of gastrointestinal bleeding with dabigatran, rivaroxaban, and warfarin: population based cohort study. Br Med J 2015; 350: h1857.

17. Seeger JD, Bykov K, Bartels DB, et al. Safety and effectiveness of dabigatran and warfarin in routine care of patients with atrial fibrillation. Thromb Haemost 2015; 114: 1277-1289.

18. Southworth MR, Reichman ME, Unger EF. Dabigatran and postmarketing reports of bleeding. New Engl J Med 2013; 368: 1272-1274.

19. Graham DJ, Reichman ME, Wernecke M, et al. Cardiovascular, Bleeding, and Mortality Risks in Elderly Medicare Patients Treated With Dabigatran or Warfarin for Nonvalvular Atrial Fibrillation. Circulation 2015; 131: 157-163.

20. Lip GY, Larsen TB, Skjoth F, et al. Indirect comparisons of new oral anticoagulant drugs for efficacy and safety when used for stroke prevention in atrial fibrillation. J Am Coll Cardiol 2012; 60: 738-746.

21. Mantha S, Ansell J. An indirect comparison of dabigatran, rivaroxaban and apixaban for atrial fibrillation. Thromb Haemost 2012; 108: 476-484.

22. Larsen TB, Skjøth F, Nielsen PB, et al.. Comparative effectiveness and safety of nonvitamin antagonist oral anticoagulants and warfarin in patients with atrial fibrillation: propensity weighted nationwide cohort study. Br Med J 2016; 350: i3189

23. Ho CW, Ho MH, Chan PH, et al. Ischaemic stroke and intracranial haemorrhage with aspirin, dabigatran, and warfarin: impact of quality of anticoagulation control. Stroke 2015; 46: 23-30.

24. Fine JP, Gray RJ. A proportional hazards model for the subdistribution of a competing risk. J Am Stat Assoc 1999; 94: 496-509.

25. Austin PC, Lee DS, Fine JP. Introduction to the analysis of survival data in the presence of competing risks. Circulation 2016; 133: 601-609.

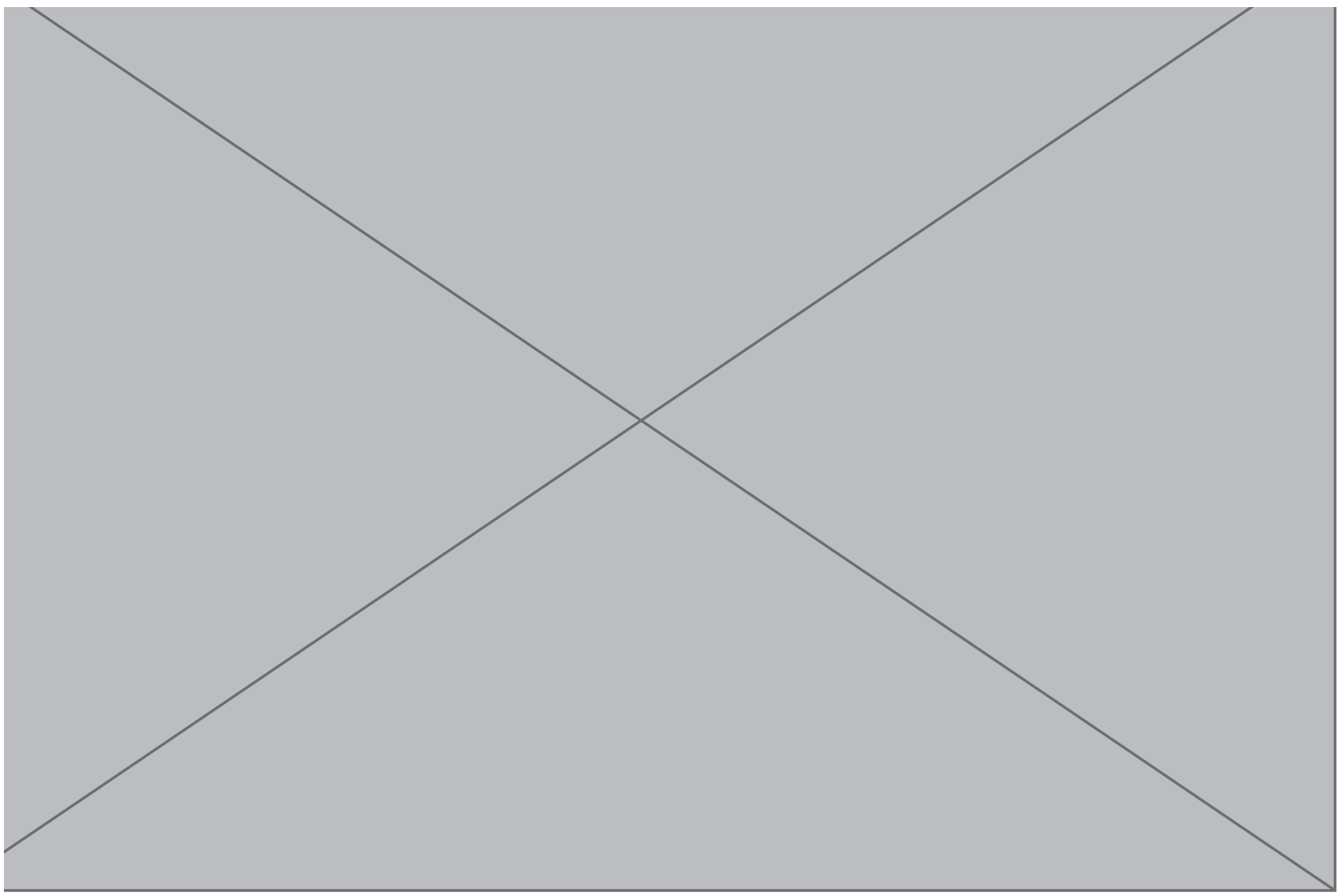

